\title{
VLBI IMAGES OF 49 RADIO SUPERNOVAE IN ARP 220
}

\author{
Colin J. Lonsdale \\ MIT Haystack Observatory, Westford, MA 01886; cjl@haystack.mit.edu \\ Philip J. Diamond and Hannah Thrall \\ Jodrell Bank Observatory, Macclesfield, SK11 9DL, UK; pdiamond@jb.man.ac.uk, hthrall@jb.man.ac.uk \\ HARDING E. SMITH \\ CASS, University of California at San Diego, 9500 Gilman Drive, La Jolla, CA 92093-0424; hsmith@ucsd.edu \\ AND \\ Carol J. Lonsdale \\ Infrared Processing and Analysis Center, MS 100-22, Pasadena, CA 91125; cjl@ipac.caltech.edu \\ Received 2006 March 6; accepted 2006 April 14
}

\begin{abstract}
We have used a very long baseline interferometry (VLBI) array at $18 \mathrm{~cm}$ wavelength to image the nucleus of the luminous IR galaxy Arp 220 at $\sim 1$ pc linear resolution, with very high sensitivity. The resulting map has an rms of $5.5 \mu \mathrm{Jy}_{\text {beam }^{-1}}$, and careful image analysis results in 49 confirmed point sources ranging in flux density from $1.2 \mathrm{mJy}$ down to $\sim 60 \mu \mathrm{Jy}$. Comparison with high-sensitivity data from 12 months earlier reveals at least four new sources. The favored interpretation of these sources is that they are radio supernovae, and if all new supernovae are detectable at this sensitivity, a resulting estimate of the supernova rate in the Arp 220 system is $4 \pm 2$ per year. The implied star formation rate is sufficient to power the entire observed far-infrared luminosity of the galaxy. The two nuclei of Arp 220 exhibit striking similarities in their radio properties, although the western nucleus is more compact, and appears to be $\sim 3$ times more luminous than the eastern nucleus. There are also some puzzling differences, and differential free-free absorption, synchrotron aging, and expansion losses may all be playing a role. Comparison with the nearby starburst galaxy M82 supports the hypothesis that the activity in Arp 220 is essentially a scaled-up version of that in M82.
\end{abstract}

Subject headings: galaxies: active — galaxies: individual (Arp 220) — galaxies: starburst — infrared: galaxies — radio continuum: galaxies — supernovae: general

\section{INTRODUCTION}

A starburst is an intense period of star formation within a galaxy that cannot be sustained over its lifetime, due to the huge amount of gas needed to fuel the process. Typical starburst lifetimes are $10^{8}-10^{9} \mathrm{yr}$. Starbursts can occur on two scales: centralized in the galactic nucleus (a nuclear starburst) or throughout the entire galaxy (a global starburst). In order to trigger a starburst, there must be a plentiful supply of gas to the center of the galaxy, which occurs when a largescale disturbance to the angular momentum of the central region causes the infall of enough material. In a global starburst this disturbance is usually associated with the collision (and possible merger) of two galaxies. In a nuclear starburst (such as M82) the disturbance may be due to the effects of shock fronts caused by a stellar bar, a tidal interaction with another galaxy, or perhaps an active galactic nucleus (AGN). The most luminous starbursts all appear to be triggered by interactions with other galaxies. Smith et al. (1998a) studied a sample of 20 radio-luminous starburst galaxies. They found that all 20 galaxies were mergers, interacting pairs, or members of groups or clusters, suggesting that all the starbursts in the sample were most likely triggered by interactions with other galaxies.

It has become clear that a "pan-spectral" approach to observations is necessary to comprehensively study starburst galaxies, combining observations taken over as large a wavelength range as possible, and supported by theoretical advances and modeling. However, particularly as the more compact and luminous nuclear starbursts are enshrouded in dust clouds that are optically thick at shorter wavelengths, long-wavelength data are uniquely able to peer through the curtain of extinction and derive the basic prop- erties of the stellar population. In some cases, IR data are sufficient, but where even IR radiation is strongly absorbed, it is necessary to turn to the millimeter and radio domains.

The IR luminosity is a widely used diagnostic of starburst activity. This strong emission comes from dust grains presumed to be heated by photons from young stars formed in the starburst. Assuming that most of the energy in photons emitted by young stars eventually results in heating the dust (which then reradiates it as blackbody thermal continuum in photons), then formation rates for stars more massive than $5 M_{\odot}$ can be estimated from the far-IR luminosity (Cram et al. 1998). Such stars dominate the total stellar luminosity, and end their lives as supernovae. Supernova rates in starbursts can be directly related to formation rates of massive stars, and by assuming an initial mass function (IMF), the luminosities of the starbursts.

Studies of radio supernovae (RSNe) in starbursts are unlike studies in our own Galaxy. The sample of RSNe in a starburst is well defined and largely free from selection effects, and all the sources can be considered to be at the same distance. Therefore, nearby starbursts can be considered as laboratories in which to investigate, both statistically and individually, the properties of a well-defined sample of RSNe and supernova remnants (SNRs).

Arp 220, at a distance of $77 \mathrm{Mpc}$ and with an infrared luminosity of $L_{\mathrm{IR}} \sim 1.3 \times 10^{12} L_{\odot}$ (Soifer et al. 1987), is one of the closest and best studied ultraluminous infrared galaxies, or ULIRGs. It is, like most ULIRGs, a merger of two gas-rich galaxies; Arp 220 has a pair of radio nuclei separated in roughly the east-west direction by approximately 370 pc (Norris 1988; Rovilos et al. 2003; Rodriguez-Rico et al. 2005). The two nuclei are also clearly visible through high-resolution Keck imaging in the mid-infrared 
(Soifer et al. 1999, 2002). The western nucleus is brighter than the eastern one, although the flux ratio is frequency dependent.

Smith et al. (1998c) made $18 \mathrm{~cm}$ continuum observations of Arp 220, in which they found a number of unresolved sources in the western nucleus, which they interpreted as luminous radio supernovae. They suggested that this interpretation is consistent with a simple starburst model for the IR luminosity of Arp 220 that has a star formation rate of 50-100 $M_{\odot} \mathrm{yr}^{-1}$ and a luminous supernova rate of $1.75-3.5 \mathrm{yr}^{-1}$. The supernovae observed are the most clearly detected RSNe in a distant galaxy. Smith et al. suggested, based on the extreme flux densities, that the objects might be Type IIn supernovae. This type of supernova is optically identified by a narrow component in its spectrum and is thought to be interacting with a dense circumstellar medium, a result of the high mass-loss rates of the progenitor stars. Rovilos et al. (2005) obtained multiepoch high-resolution data of Arp 220, and concluded that the light curves had been surprisingly stable over a period of more than $5 \mathrm{yr}$. The long decay time of the $18 \mathrm{~cm}$ light curves of these sources does not agree with the simple model of Type IIn supernovae (Smith et al. 1998c). The observations discussed in this paper are part of a subsequent high-sensitivity monitoring program of Arp 220.

\section{OBSERVATIONS AND DATA REDUCTION}

We observed Arp 220 on 2003 November 9 with a global array of the largest telescopes on Earth. The observations were part of a long-term monitoring campaign aimed at studying the changes in the compact structure of both the continuum and OH maser emission in Arp 220. Eighteen antennas took part in the experiment, including the 10 VLBA antennas, the phased Very Large Array (VLA), the Green Bank Telescope (GBT), Arecibo, and five antennas of the European VLBI Network (EVN). All antennas performed satisfactorily except for the phased VLA; it was unable to maintain phase coherence due to the presence of interference, and so all phased-VLA data were flagged.

The observations covered a period of almost $21 \mathrm{hr}$. Data were taken in two polarizations and in four $8 \mathrm{MHz}$ wide intermediate frequency (IF) bands covering the frequency range 1633.991665.99 MHz and with an aggregate data rate of $256 \mathrm{Mbit} \mathrm{s}^{-1}$. The strong redshifted $\mathrm{OH}$ maser emission from Arp 220 was centered in the lowest of the four IF bands. The line-free portions of the lowest IF, plus the upper $24 \mathrm{MHz}$ from the other $3 \mathrm{IFs}$, were dedicated to observing the continuum emission from Arp 220. The data were recorded on tape and shipped to the VLBA correlator in Socorro, New Mexico. There, two correlator passes were done, one with 512 channels across the $8 \mathrm{MHz}$ of the bottom IF containing the $\mathrm{OH}$ line, and a second with 32 channels per IF across all four IFs. The correlated data were sent to Jodrell Bank Observatory for subsequent data processing.

Due to the presence of strong and compact $\mathrm{OH}$ maser emission (Lonsdale et al. 1994, 1998), which could be used for phase calibration, there was no need for phase referencing. Therefore, the observing scheme was straightforward, with most time being spent on Arp 220 and with occasional 15-minute scans on calibrator sources for the purpose of tracking the residual delays and determining the bandpass corrections. The calibrator sources used for this purpose were $1613+341$ and $1516+193$. The J2000 coordinates assumed for Arp 220 were R.A. $=15^{\mathrm{h}} 34^{\mathrm{m}} 57.225^{\mathrm{s}}$, decl. $=23^{\circ} 30^{\prime} 11^{\prime \prime} .564$.

All data calibration was carried out using the 15DEC01 release of NRAO's AIPS software and used standard procedures (Diamond 1995). The data were corrected for digital effects and those of parallactic angle rotation; the gross residual delay errors in each observing band and the phase offsets between the bands were determined; amplitude calibration was performed using the system temperature and gain curves supplied by the observatories; any time-variable residual delay offsets and the full complex bandpass solution for each antenna were determined; the residual antenna-based phase delay rates of the strongest channel in the $\mathrm{OH}$ spectrum was measured at 5 minute time intervals. All of these calibration factors were then applied to the data to produce a data set coherent in time and frequency. Then, an iterative loop of self-calibration and imaging was performed on the $\mathrm{OH}$ reference channel to determine and correct for the short-timescale (1 minute) antenna-based phase errors; the corrections thus derived were applied to all the frequency bands, thereby ensuring that the positional registration of the continuum emission, when imaged, was anchored to the position of the peak of the strong line emission.

The continuum emission in Arp 220 is problematic from a VLBI imaging perspective in that the structure has a very different character depending on the spatial resolution with which it is viewed. As we have previously shown with MERLIN (Rovilos et al. 2003), on arcsecond scales we see all of the expected $\sim 280$ mJy flux from Arp 220 (Baan et al. 1982). About $200 \mathrm{mJy}$ of this is concentrated in two main components separated by $1^{\prime \prime}$ almost eastwest, which are surrounded by more extended emission. However, our early VLBI observations (Smith et al. 1998c) showed that on baselines beyond a few hundred kilometers, most of the MERLINscale structure was resolved and we only see a few percent of the flux concentrated in a number of point sources. Therefore, in producing the continuum image from this data set, which suffers from poor $(u, v)$ coverage on short baselines, we must take care when imaging. We found that the best approach was to simply eliminate the short baselines and the diffuse emission detected on them, by ignoring all data within a $(u, v)$-range of $5000 \mathrm{k} \lambda$, i.e., projected baseline lengths of less than $900 \mathrm{~km}$. This results in only minimal loss of sensitivity to compact features, because the largest apertures in the telescope array tend to be at the periphery of the array, and tend not to participate in many short baselines.

Regions of the final image, centered on the two nuclei of Arp 220, are shown in Figure 1. The rms noise across the image varies somewhat with location due to dynamic range limitations in areas with a high density of compact sources. However, we find that in the emptiest areas of the image the rms noise is $5.5 \mu \mathrm{Jy}_{\text {beam }}{ }^{-1}$, and close to the northwest region of compact structure it approaches $9 \mu \mathrm{Jy}$ beam $^{-1}$ : this image is, therefore, the most sensitive VLBI image obtained to date by some margin. Many point sources are clearly visible. Due to the variable noise levels and the large number of faint source candidates, objective criteria for identifying and measuring sources were required, as described in the next section.

\section{IMAGE ANALYSIS AND RESULTS}

The focus of this investigation is the population of point sources in the two nuclei of Arp 220. For a reliable and complete source list, we need objective and robust criteria for source detection. A careful treatment is required, because the deconvolution step of image generation, via the CLEAN algorithm, is highly nonlinear in nature, contributing to strongly non-Gaussian noise statistics in the final image; another factor that may influence the statistics is residual poorly constrained diffuse-continuum emission. The statistic we need to develop for each candidate source is a probability of false detection, $\Psi$, determined empirically from analysis of noise statistics as a function of position within the image.

As noted above, the rms fluctuation level varies across the image. Rms values have been measured in the immediate vicinity of each source candidate $\mathrm{C}$, which we refer to as local variances and denote by $\sigma_{C}$. For a measured flux density, $S_{C}$, of a candidate source, we define an effective signal-to-noise ratio $\eta$, such that 


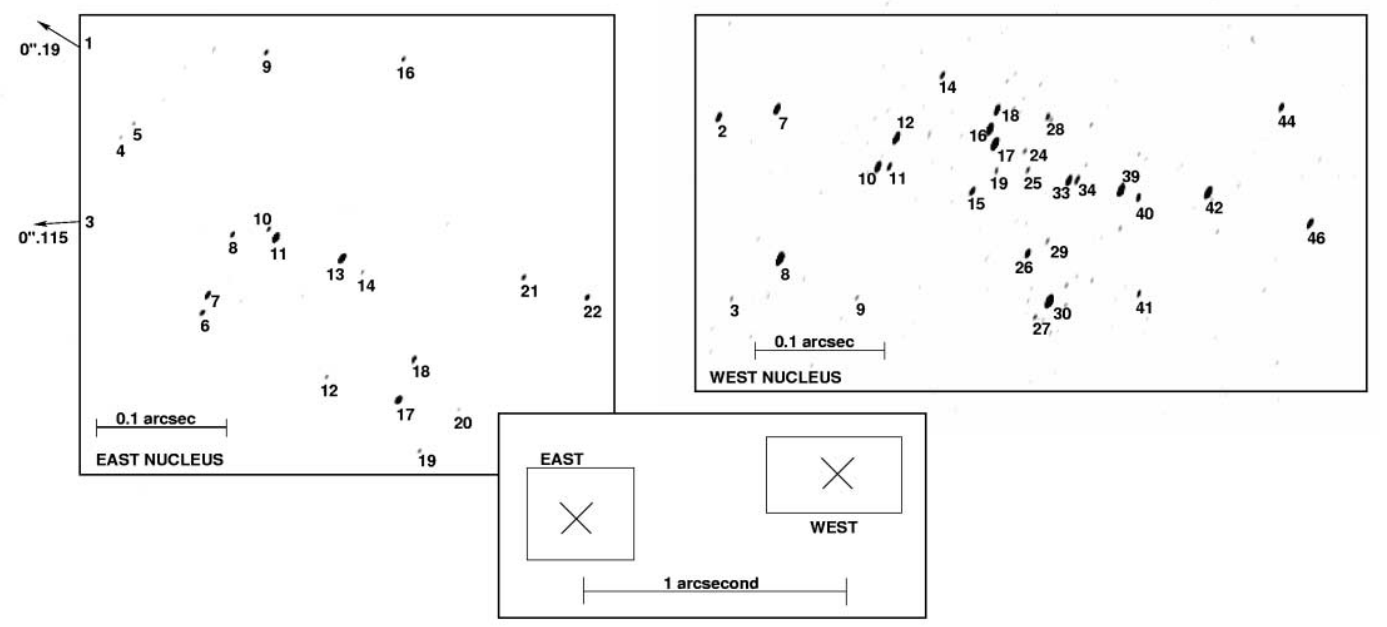

FIG. 1.-Continuum images of the east and west nuclei of Arp 220. The angular resolution is $5.9 \times 2.7$ mas in position angle $-21^{\circ}$. The background rms level in the east nucleus ranges from 5 to $6 \mu \mathrm{Jy} \mathrm{beam}^{-1}$, and in the west nucleus ranges from 7 to $9 \mu \mathrm{Jy}$ beam ${ }^{-1}$. Sources which meet the detection criteria (see text) are numbered separately for east and west nuclei, and are identified in Table 1 as En and $\mathrm{W} n$ respectively, where $n$ is the source number. The inset shows the relative locations of the two images, and the crosses mark the relative locations of the peaks of the diffuse radio continuum emission in the two nuclei, as measured using MERLIN at $6 \mathrm{~cm}$ (Fig. 2 of Rovilos et al. 2003). It can be seen that the point source locations are highly correlated with the diffuse continuum distribution. The diffuse emission, which is 20 times stronger than the sum of the point source fluxes, is fully resolved out by our VLBI array.

$S_{C}=\eta \sigma_{C}$. We can then estimate the probability of false detection, $\Psi_{C}$, for source candidate $\mathrm{C}$ by reference to an empirically determined $\Psi(\eta)$. Construction of our source list is then accomplished by measuring all candidates down to some low flux density level, and then applying a cutoff threshold in $\Psi_{C}$ (which is equivalent to a threshold in $\eta$ ).

In order to determine $\Psi(\eta)$ in a manner that is representative of the image areas in which candidate sources are being measured, we removed all source candidates from the image, along with the few areas immediately adjacent to brighter sources that suffer from obvious sidelobe artifacts. We then selected two areas, one enclosing the eastern nucleus and one the western nucleus. We also analyzed a comparison region between the two nuclei. Histograms of the candidate-free pixel values were constructed, and probability distributions of local rms levels were derived (Fig. 2). It can be seen that despite the factor of 1.4 difference in rms levels between east and west, the shape of the probability distributions is very similar in the two nuclei, and quite similar in the comparison region. All three distributions are also strongly non-Gaussian, as illustrated in the diagram. While we show only positive pixel values here, the distributions are closely symmetric around zero, which implies that the departure from Gaussian statistics is not due to a population of weak sources below the detection limit, and is instead a product of the image reconstruction algorithms.

We have extrapolated the probability function for the regions enclosing the two nuclei via a least-squares linear fit to the data from both regions (dashed line). We have then used this fit to estimate $\Psi(\eta)$.

Note that pixels on the image are not independent on the angular scale of the array resolution. In addition, the observed strong clustering of candidate sources around the two nuclei defines a limited region of sky over which our search for sources is to be conducted. Therefore, while the full image contains $\sim 2$ million pixels, the search is conducted over only $\sim 2 \times 10^{4}$ independent resolution elements. It is the probability of false detection within this number of independent image samples to which $\Psi(\eta)$ must refer.

For a given candidate source with $\mathrm{S} / \mathrm{N} \eta_{C}$ (i.e., flux density divided by local $\mathrm{rms}$ ), the probability that any given pixel value $\eta_{P}$ is less than this is given by $P\left(\eta_{P}<\eta_{C}\right)=1-P\left(\eta_{P}>\eta_{C}\right)$, where $\log \left[P\left(\eta_{P}>\eta_{C}\right)\right]$ is the quantity on the vertical axis of Figure 2. By using a least-squares linear fit to the data from the two nuclei displayed in Figure 2, we model this function as $\log \left[P\left(\eta_{P}>\right.\right.$ $\left.\left.\eta_{C}\right)\right]=-0.446 \eta_{C}-0.788$, denoted by the dashed line. The probability that all pixels searched are below $\eta_{C}$ is $\left[1-P\left(\eta_{P}>\eta_{C}\right)\right]^{n}$, where $n$ is the number of independent resolution elements searched $\left(2 \times 10^{4}\right.$ in this case), and we have

$$
\log \left(1-\Psi_{C}\right)=n \log \left[1-P\left(\eta_{P}>\eta_{C}\right)\right]
$$

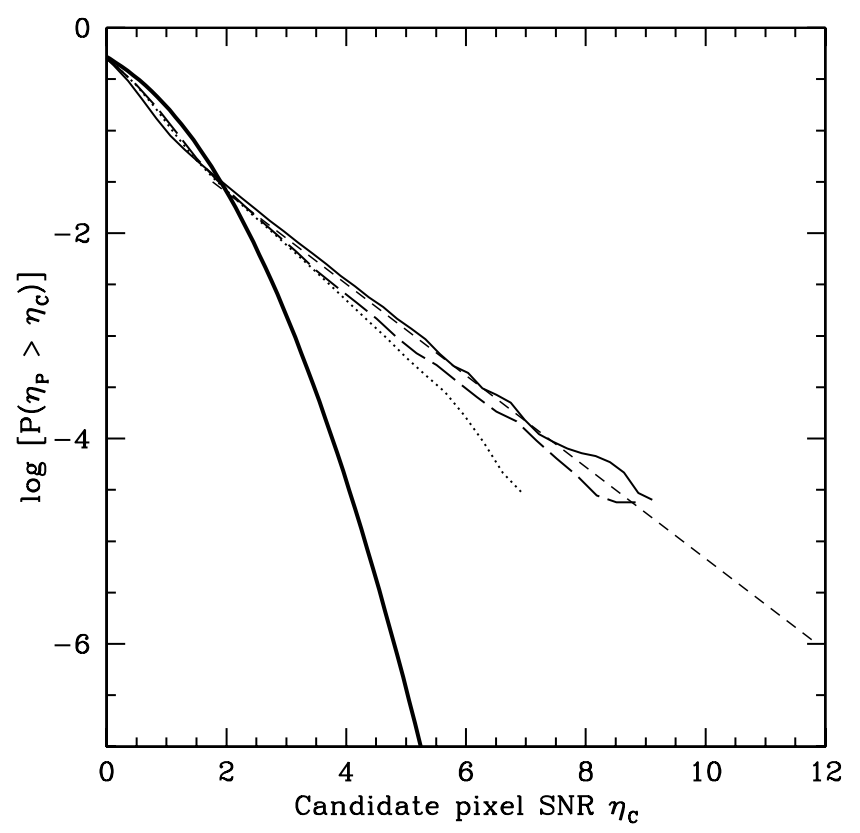

FIG. 2.- Noise statistics for various regions of the Arp 220 image. The horizontal axis is $\mathrm{S} / \mathrm{N}$ of a candidate pixel, $\eta_{C}$, defined as the measured value divided by the local rms. The vertical axis gives the probability that a randomly selected pixel of $\mathrm{S} / \mathrm{N} \eta_{P}$ will exceed a given candidate pixel $\mathrm{S} / \mathrm{N} \eta_{C}$, based on the statistics of the image in a region. The long-dashed curve refers to the eastern nucleus, the solid curve to the western nucleus, and the dotted curve to a region between the two nuclei. The short-dashed straight line is a linear least-squares fit to the curves for the east and west nuclei, and is the basis for extrapolation to observed $\mathrm{S} / \mathrm{N}$ values of source candidates, and thence to estimates of the probability of false detection on a case-by-case basis (see text). The heavy black curve represents a Gaussian distribution of pixel values. 
TABLE 1

Point Source Candidates in Arp 220

\begin{tabular}{|c|c|c|c|c|c|c|c|c|c|}
\hline Name & $\begin{array}{l}\delta \text { R.A. } \\
\text { (mas) }\end{array}$ & $\begin{array}{c}\delta \text { Decl. } \\
\text { (mas) }\end{array}$ & $\begin{array}{l}\text { Flux } \\
(\mu \mathrm{Jy})\end{array}$ & $\Psi_{C}$ & Name & $\begin{array}{l}\delta \text { R.A. } \\
\text { (mas) }\end{array}$ & $\begin{array}{c}\delta \text { Decl. } \\
\text { (mas) }\end{array}$ & $\begin{array}{l}\text { Flux } \\
(\mu \mathrm{Jy})\end{array}$ & $\Psi_{C}$ \\
\hline E1 ............................. & 658.3 & 225.3 & 69.7 & 0.04 & 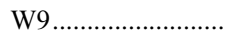 & -422.3 & 50.0 & 74.5 & 0.00 \\
\hline 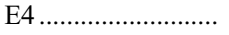 & 496.9 & 56.1 & 52.9 & 0.00 & 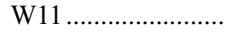 & -447.2 & 150.9 & 175.4 & 0.00 \\
\hline 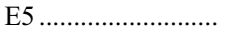 & 488.3 & 67.3 & 57.3 & 0.14 & 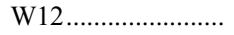 & -452.8 & 173.0 & 645.6 & 0.00 \\
\hline 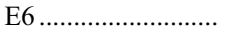 & 442.3 & -87.3 & 90.8 & 0.00 & 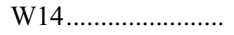 & -487.6 & 221.2 & 135.9 & 0.00 \\
\hline E9 .......................... & 399.5 & 125.6 & 81.1 & 0.00 & 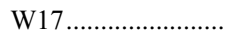 & -527.7 & 168.4 & 717.4 & 0.00 \\
\hline 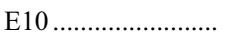 & 397.8 & -19.0 & 80.0 & 0.01 & 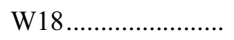 & -529.2 & 194.4 & 255.1 & 0.00 \\
\hline 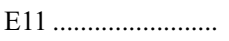 & 392.9 & -26.0 & 296.3 & 0.00 & W19 & -528.8 & 147.5 & 95.5 & 0.10 \\
\hline 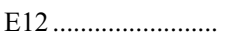 & 358.8 & -139.8 & 65.4 & 0.03 & 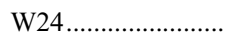 & -550.6 & 163.0 & 90.0 & 0.06 \\
\hline 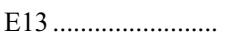 & 348.7 & -43.0 & 234.8 & 0.00 & 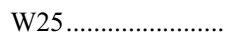 & -552.9 & 148.2 & 98.0 & 0.01 \\
\hline 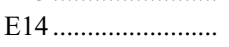 & 334.8 & -54.2 & 62.1 & 0.09 & 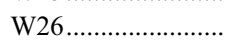 & -552.6 & 83.9 & 219.8 & 0.00 \\
\hline 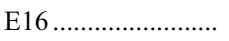 & 307.2 & 120.1 & 79.6 & 0.00 & 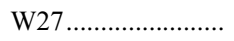 & -558.2 & 35.1 & 96.4 & 0.03 \\
\hline 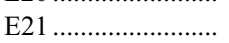 & 226.4 & -58.3 & 86.0 & 0.00 & 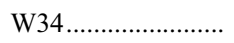 & -590.6 & 140.7 & 160.8 & 0.00 \\
\hline \multirow[t]{2}{*}{ 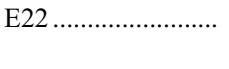 } & 183.9 & -74.8 & 97.4 & 0.00 & 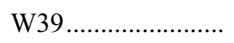 & -624.0 & 133.0 & 870.2 & 0.00 \\
\hline & & & & & 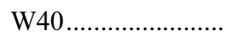 & -637.0 & 127.1 & 195.5 & 0.00 \\
\hline 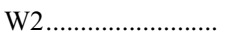 & -317.0 & 189.0 & 253.6 & 0.00 &  & -637.5 & 53.2 & 111.7 & 0.00 \\
\hline 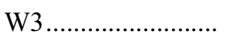 & -327.0 & 49.4 & 75.3 & 0.04 & 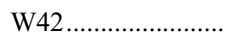 & -690.5 & 131.0 & 713.5 & 0.00 \\
\hline 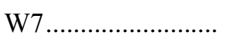 & -361.6 & 195.3 & 368.8 & 0.00 & 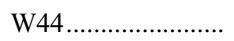 & -746.2 & 196.6 & 207.5 & 0.00 \\
\hline 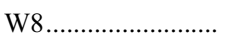 & -363.8 & 79.8 & 849.3 & 0.00 & 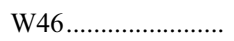 & -768.0 & 107.2 & 271.2 & 0.00 \\
\hline
\end{tabular}

Note- Table 1. Source candidates and measured properties in rough right ascension order. A source candidate is included in the table if its flux density exceeds 9.5 times the local rms of the background (with the exceptions of E4 and W9 as discussed in the text). The fifth column lists the probability of false detection $\left(\Psi_{C}\right)$ of each candidate based on analysis of the noise statistics of the image, as defined in the text. The sum of these probabilities is such that the expectation for the number of false positive detections is $<1$, out of this list of 49 candidates.

and the total expected number of false detections is then given by

$$
N_{\text {false }} \sim \sum_{C} \Psi_{C}
$$

In order to achieve $N_{\text {false }}<1$, we find that we must omit candidate sources with $\eta_{C}<9.5$. The result is a list of 47 sources, with the expectation of $<1$ false positive. There are also 4 candidate sources with $9<\eta_{C}<9.5$, of which 2 have faint but unmistakable counterparts on an image from 12 months earlier (not yet published), and in which we therefore have high confidence. Our final source list thus contains 49 objects, listed in Table 1, which are the basis for the ensuing discussion.

We have adopted a naming convention of En and $\mathrm{W} n$ for sources in the east and west nuclei respectively, where $n$ is a sequential candidate number, arbitrarily in order of decreasing right ascension. Not all candidates met our detection criteria, so the source numbers in Table 1 are not sequential. In future papers, newly discovered sources will be assigned higher sequential numbers.

As a footnote from this analysis of image statistics, it is an interesting fact that in all three regions analyzed (east nucleus, west nucleus, and control region), the data values below a $\mathrm{S} / \mathrm{N}$ of $\sim 1$ closely follow a Gaussian distribution, with equivalent rms values just below $5 \mu \mathrm{Jy}_{\text {beam }}{ }^{-1}$ in all three cases. The location-dependent departures from Gaussian distributions represent a high kurtosis parameter, and a "leptokurtic" distribution. The consistency of the low-S/N Gaussian fits strongly suggests that the underlying thermal noise level on the map is $\sim 4.8 \mu \mathrm{Jy} \mathrm{beam}^{-1}$, and that the rest is sidelobe noise propagating through the complex, nonlinear imaging process. Our results may provide a cautionary note to others seeking to interpret low-level features in similar images.

\section{DISCUSSION}

\subsection{General Observations}

Figure 1 shows the overall structure of the continuum emission from Arp 220 as observed with VLBI. The sensitivity level achieved in this image is significantly better than earlier efforts (Smith et al. 1998c; Rovilos et al. 2003), and represents the current state of the art in high-sensitivity radio imaging. As a result of the sharply reduced noise levels, we have been able to detect 49 continuum point sources in Arp 220 (29 in the west nucleus, and 20 in the east). From the current observations, we note the following pertinent features of the structure:

1. All 49 sources appear unresolved to our array, and typical upper limits on their linear extent are less than $1 \mathrm{pc}$.

2. The sources are clustered, with 22 of the 29 western sources falling within a $0.25 \times 0.15$ region, and 14 of the 20 eastern sources falling within a $0.3 \times 0.2$ region. In both nuclei, sources falling outside these compact regions tend to be weaker.

3. The total flux density accounted for in the point sources is $2.0 \mathrm{mJy}$ in the east and $9.8 \mathrm{mJy}$ in the west. The total $18 \mathrm{~cm}$ continuum flux densities of the two nuclei, for comparison, are 92 and $111 \mathrm{mJy}$ respectively, with another $\sim 80 \mathrm{mJy}$ on larger scales (Rovilos et al. 2003). The VLBI-scale emission accounts for only a few percent of the total.

4. The diffuse emission, which dominates the total $18 \mathrm{~cm}$ emission from Arp 220, is generally resolved out by our array (but see discussion in $\S 2$ ). Based on the clustering, we estimate that the 
Rovilos et al. (2003) MERLIN peak-flux density may have up to a $15 \%$ contribution from the compact sources in the west, and less than $2 \%$ in the east.

5. Sources in the east nucleus are systematically weaker than those in the west. Based on the expanded size of the sample now available, this is a robust result. The apparent luminosity function of the sources is markedly different between the two nuclei.

6. The source distributions in the two nuclei mirror the properties of the diffuse emission, namely stronger and more compact in the west, weaker and less compact in the east. Furthermore, the centroids of the source clusters in west and east closely match the relative locations of the two nuclei as seen at $6 \mathrm{~cm}$ by MERLIN (see Fig. 1). The properties of the point sources and of the diffuse emission are strongly correlated.

7. Consistent with earlier results, none of the point sources detected here is positionally coincident with the strong, compact $\mathrm{OH}$ maser features in Arp 220 (However, several sources show both $\mathrm{OH}$ absorption and low-gain maser amplification; results in preparation.)

We can also compare the current results to earlier data. The most relevant comparison at this time is to data taken in experiment GL26B, 12 months earlier than the current GD17A observations. The GL26B data have presented challenging calibration problems, but an image of sufficient quality for meaningful comparison, with rms noise of $\sim 9 \mu \mathrm{Jy}$, is available. We find that in the intervening 12 months, at least 4 new sources have appeared, namely E8, W11, W25 and W34. Their flux densities in the current image are such that they would have been readily detected in the GL26B data. Source W40 is also possibly new, but the evidence is less compelling. Another, bright source (W12), was present 12 months earlier, but conspicuously absent 3.4 yr earlier (by comparison with data from Rovilos et al. [2005]).

It is premature to derive new, high-sensitivity light curve data for a large number of sources. The necessary monitoring data have been taken for multiple epochs, but the analysis is complex and time consuming, and accurate flux scale registration requires additional work. These results, including the GL26B data referred to above, will be presented separately.

\subsection{Radio Supernovae and the Nuclear Starburst}

The original interpretation of the point sources in Arp 220 as radio supernovae (RSNe) by Smith et al. (1998c) remains the favored one. The sources are unresolved, and exhibit flux density variations on timescales of months and years (Rovilos et al. 2005). Several new sources have been observed to appear where none previously existed. The observed flux densities, between 50 and $1200 \mu \mathrm{Jy}$, correspond to $1.6 \mathrm{GHz}$ radio powers between $3.5 \times 10^{19}$ and $8.3 \times 10^{20} \mathrm{~W} \mathrm{~Hz}^{-1}$, which falls in the upper end of the range of observed peak powers for known RSNe (van Dyk et al. 2000).

The widespread variability and frequent appearance of new sources constitutes compelling evidence that a significant fraction of the sources are young and compact. Is there, however, a possibility that some of the sources are much older, and would be more conventionally classified as supernova remnants (SNRs)? There are, however, no known examples of decades or centuries-old SNRs with radio luminosities approaching those of the sources in Arp 220. The faintest detected sources in Arp 220 are of order 50 times more luminous than the brightest Galactic SNRs with known explosion dates (e.g., Cassiopeia A), and typically an order of magnitude more luminous than the bright resolved RSN in the nearby starburst galaxy M82 (see $\S 4.3$ ). The most plausible interpretation is that all the detectable sources in Arp 220 are young, and consequently luminous. Given the apparent rate of

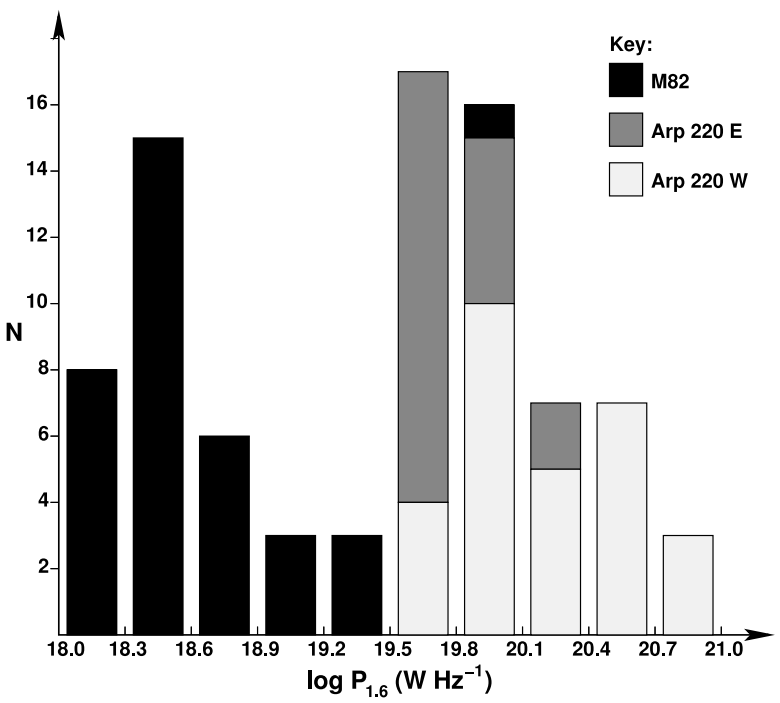

FIG. 3.- Histogram of the $1.6 \mathrm{GHz}$ monochromatic power for compact sources in M82 and Arp 220. There are two important features illustrated here. First, the M82 sources are almost all weaker than those detected in Arp 220, with typical Arp 220 sources being several tens of times stronger than typical M82 sources. The exception to this is the source $41.95+57.5$ in M82, which is declining at $8.5 \% \mathrm{yr}^{-1}$ (Kronberg et al. 2000). Second, the power distributions of sources in the E and W nuclei of Arp 220 are markedly different. Not only are the eastern sources weaker on average, but the shape of the distribution appears more strongly biased toward weaker sources. The M82 data are taken from Wills et al. (1998), Allen and Kronberg (1998), and private communication from R. Beswick. The Arp 220 data are from this paper.

appearance of new sources, and the total number of sources detected, the turnover rate of sources in a uniform population would imply that the oldest objects are likely to be at most a few decades old. However, the population is clearly heterogeneous, and there is evidence that the brighter sources tend to decay slowly. More information is needed in order to reliably estimate ages.

Recently, our team has detected several of the point sources at $2.3,5$, and $8.4 \mathrm{GHz}$ (J. E. Conway et al., in preparation); preliminary analysis indicates that they exhibit a range of spectral indices, from flat to steep. Further analysis and interpretation will be provided in the forthcoming paper. In the absence of external free-free absorption, RSNe that are no longer in the rising phase at $18 \mathrm{~cm}$ are expected to exhibit steep spectra characteristic of optically thin synchrotron emission. There is considerable evidence, however, for significant free-free optical depths at $18 \mathrm{~cm}$ in the nuclei of powerful ULIRGs (e.g., Condon et al. 1991), which would likely manifest itself as spatially correlated flattening of RSN spectra across regions of the Arp 220 nuclei. Any interpretation of the star-forming environment in the nuclei, based on our $18 \mathrm{~cm}$-only RSN measurements, must take into account the possibility that radio luminosities, RSN counts, and rates of new source appearances may all be underestimated.

\subsubsection{Differing RSN Populations in East and West}

The two nuclei of Arp 220, although strikingly similar in many properties (numerous RSN, strong compact $\mathrm{OH}$ megamaser emission, strong diffuse continuum emission), display differing properties with regard to the point sources. The two principal differences are that (1) the point sources are systematically stronger in the west (i.e., the luminosity functions are different as can be seen in Fig. 3); and, (2) the sources are confined to a somewhat morecompact region in the west. We can quantify the difference in luminosity functions by applying a Kolmogorov-Smirnov test to the flux density data. A simple comparison yields a probability 
of 0.001 that the samples are drawn from the same population, primarily due to the much higher mean flux density in the west.

An obvious hypothesis to explain this result is that the eastern nucleus population is just a fainter replica of that in the west. A simple model relating expected RSN luminosities to the far-infrared luminosity can be constructed, using a $t^{-\gamma}$ decay profile where $\gamma=1.3$ (Smith et al. 1998b, 1998c), and translating $L_{\mathrm{FIR}}$ into a relative star formation rate and hence a supernova rate. This leads to mean RSN ages proportional to $L_{\mathrm{FIR}}^{-1}$, and RSN fluxes $S_{\mathrm{RSN}} \propto$ $L_{\mathrm{FIR}}^{\gamma}$. The fainter sources in the east would then simply reflect a less-luminous starburst than in the west, which is qualitatively consistent with the less-compact morphology. Note that such a model cannot modify the shape of the RSN luminosity function, but can only translate it to lower luminosities as the starburst intensity and associated $L_{\mathrm{FIR}}$ declines.

To test this, we can truncate the western source list to the brightest 20 objects, and scale the fluxes to match the effective sensitivity limit in the eastern nucleus reduction by a factor of 2.7. Comparing the resulting distributions, the $\mathrm{K}-\mathrm{S}$ test indicates a probability of $\sim 13 \%$ that the samples are drawn from the same population - the populations still differ, although the confidence level is not high. It appears that a simple model in which the western nucleus hosts a higher star formation rate than the eastern nucleus, for whatever reason, may not adequately predict the observed point source properties. This suggests that other factors may be involved, such as differing chemical compositions and IMFs between the nuclei resulting in a different mix of supernova types, differing ISM densities influencing the RSN light curves, or a time dependence in the exponent $\gamma$ of unknown origin.

\subsubsection{Comparison with SN 2000ft in NGC7469}

Recently, a luminous RSN has been reported in the ULIRG NGC7469 (Colina et al. 2001; Alberdi et al. 2006). This source, SN 2000ft, exhibits a well-determined classic Type II RSN light curve, with an accurately modeled explosion date and a relatively steep flux density decay $(\gamma=1.8)$. NGC 7469 is at a distance similar to Arp 220, and SN 2000ft reaches modeled peak 8, 5, and $1.6 \mathrm{GHz}$ flux densities of $2,1.5$, and $0.35 \mathrm{mJy}$, respectively. In Arp 220, this supernova would thus have appeared at $1.6 \mathrm{GHz}$ as a moderately bright but very short-lived RSN among a population that is typically much longer lived. Alberdi et al. argue that the classical behavior of the RSN indicates a light curve governed by ejecta interacting with the pre-existing stellar wind (e.g., Weiler et al. 1986). They predict that when the ejecta reach the dense ISM presumed to surround the supernova site, the light curve decay rate will slow dramatically.

Clearly, many of the RSN in Arp 220 are behaving differently, and the slow decay (ISM interaction) phase appears to have been reached while the radio luminosity is still high. This would be a natural consequence if these objects are embedded in a denser ISM than SN 2000ft, so that the ISM interaction phase occurs earlier. Such may commonly be the case for RSN in the compact nuclei of Arp 220, which are likely to have a higher mean density than the $1 \mathrm{kpc}-$ scale circumnuclear starburst region of NGC 7469.

This comparison leads to the expectation of a range of behaviors within the Arp 220 RSN population, reflecting ISM inhomogeneity, and a range in the strength and onset timing of the ISM interaction phase. Heterogeneity in the RSN population renders efforts to constrain the supernova rate by examination of luminosity functions and light curves unattractive. The supernova rate is instead best determined directly via sufficiently sensitive and frequent monitoring for new sources. It can then be used as a powerful constraint on models in order to understand the evolution of the population.

\subsubsection{Supernova Rate and Starburst Luminosity}

By virtue of a severalfold improvement in sensitivity and RSN detection threshold, we have directly observed 4 new sources in a 12-month period, with three in the western nucleus and one in the eastern nucleus. We do not know whether all Type II supernovae in Arp 220 maintain detectable flux density levels within 12 months of initial appearance, but if so, we have a measure of the supernova rate in the galaxy at $4 \pm 2$ per year. If not, we may have missed some, and this becomes a lower limit.

Smith et al. (1998b, 1998c) discuss the relationship between FIR luminosity, star formation rate, the stellar IMF, and the supernova rate. They conclude that based on the luminosity of Arp 220, a supernova rate of $2 \mathrm{yr}^{-1}$ can be expected, with an uncertainty of about a factor of 2 depending primarily on assumptions about the IMF. Our estimate of $4 \pm 2 \mathrm{yr}^{-1}$ based on the current observations is consistent with the Smith et al. estimate. Clearly, the starburst traced by these RSNe is capable of generating the entire FIR luminosity of Arp 220, within the uncertainties of our measurements and of starburst modeling. A hidden AGN, while not ruled out by these observations, is not required on energetic grounds.

A high star formation and supernova rate in Arp 220 would be expected to generate a large X-ray luminosity from X-ray binaries (XRBs). A relationship between starburst-powered FIR luminosity and X-ray luminosity has been established (e.g., Persic et al. 2004), but Arp 220 does not fall on the correlation, being underluminous in X-rays. This could be explained by extreme absorption column densities, or by relative youth of the starburst compared to XRB lifetimes resulting in a deficiency of XRBs. Iwasawa et al. (2005) report XMM-Newton detection of prominent Fe $\mathrm{K} \alpha$ line emission, throwing into doubt the interpretation of the weak hard $\mathrm{X}$-ray emission in Arp 220 as originating from X-ray binaries, and further exacerbating the problem. They appealed to the possibility of a low supernova rate and an energetically dominant buried AGN to explain their findings. The current work instead strongly confirms a high supernova rate, so the $\mathrm{X}$-ray binary issue remains problematic. A robust independent estimate of typical absorption columns to the starbursting regions of Arp 220 is needed, and the measurement of free-free optical depths through VLBI techniques will be valuable in this regard.

\subsubsection{West-East Luminosity Ratio}

An important question concerns the ratio of luminosities between the two nuclei. Using $25 \mu \mathrm{m}$ imaging, Soifer et al. (1999) measured a 3:1 ratio, with the western nucleus being the stronger. Since most of the luminosity of Arp 220 emerges at longer wavelengths (around $100 \mu \mathrm{m}$ ), and since there is evidence for significant opacity at $25 \mu \mathrm{m}$ (Soifer et al. 2002), the infrared evidence strongly suggests a $3: 1$ luminosity ratio, but is not conclusive, and does not dictate an origin (AGN or starburst) for the luminosity.

The RSN data presented here support a roughly $3: 1$ ratio of starburst luminosities between the nuclei. The different rates in the east and the west $\left(1 \mathrm{yr}^{-1}\right.$ and $3 \mathrm{yr}^{-1}$ respectively), while obviously very poorly determined due to small number statistics, are roughly consistent with ratio of luminosities (2.7) assumed in $\S 4.2 .1$ for testing the hypothesis that the eastern nucleus differs from the west only in starburst intensity and typical RSN ages. Future monitoring will quickly refine this statistical result.

The apparent agreement between infrared and RSN evidence for a 3:1 ratio lends weight to the hypothesis that the bolometric luminosity of Arp 220 is dominated by starburst activity. If AGN 
activity were a major contributor, one would expect the overall ratio to differ from the ratio based solely on starburst activity. Although requiring confirmation with more data unaffected by opacity, this appears not to be the case; starburst activity traced by radio supernovae yields the same luminosity ratio as a method (IR imaging) which is blind to the luminosity source.

\subsubsection{The Diffuse Radio Continuum}

A supernova origin for the synchrotron plasma responsible for the diffuse continuum emission is strongly supported by our data. We see a detailed correspondence between the properties of the supernova aggregations and the diffuse emission regions, consistent with diffusion of supernova remnant relativistic electrons into the interstellar medium. The degree of correspondence suggests that the electrons are not diffusing very far, most likely due to relatively short synchrotron lifetimes of the electrons. The ISM density could also be involved, since it may influence both the local star formation rate and the local synchrotron emissivity when seeded with SNR electrons.

This emission is of the kind expected to conform to the wellknown FIR/radio correlation, and indeed it has been shown that ULIRGs in general follow this correlation, after correction for freefree absorption (Condon et al. 1991). We therefore expect an underlying bolometric luminosity ratio of $3: 1$ between the nuclei to be reflected in a similar ratio of diffuse continuum fluxes. This does not appear to be the case; at $18 \mathrm{~cm}$ the diffuse continuum flux ratio between the two nuclei is only $\sim 1.2$ (Rovilos et al. 2003). Further, Rodriguez-Rico et al. (2005) measure a ratio of $\sim 1.25$ at both 8.4 and $43 \mathrm{GHz}$. A ratio of closer to $2: 1$ at the emission peaks is indicated from observations at higher angular resolution by Rovilos et al. (2003) at $5 \mathrm{GHz}$, and by Norris (1988) at 5, 15, and $22 \mathrm{GHz}$.

This apparent discrepancy can be understood through a combination of differential free-free absorption at $1.6 \mathrm{GHz}$, and differential energy loss rates in the synchrotron plasma. The western nucleus is stronger and more compact than the eastern nucleus. In fact, in our 1.6 GHz VLBI observations, the diffuse emission from the western nucleus is sufficiently compact at its peak that it is strongly detected on our shortest baselines, which must be excised in order to preserve imaging fidelity of the RSN (see $\S 2$ ). The diffuse continuum brightness distribution is strongly peaked, and is poorly modeled by a Gaussian. This compactness of the peak will be associated with higher free-free optical depths, and higher energy densities and pressures which will plausibly lead to more rapid expansion losses in the synchrotron plasma.

Regarding free-free absorption, following Smith et al. (1998b) the mean absorption optical depth is likely to be $\sim 0.5$ in Arp 220, and certainly higher in the inner regions of the western nucleus. This is adequate to explain the $1.6 \mathrm{GHz}$ result of Rovilos et al. (2003).

To explain the low radio flux ratio at higher frequencies where free-free absorption is negligible, we must consider the properties and evolution of the diffuse synchrotron plasma. It is noteworthy that the observed diffuse radio properties of the Arp 220 nuclei yield lifetimes for electrons radiating at $\sim 5 \mathrm{GHz}$ of between $10^{4}$ and $10^{5} \mathrm{yr}$ (assuming field/particle energy equipartition). These times are short compared to typical starburst lifetimes of $10^{7} \mathrm{yr}$ or more, so in the absence of other energy-loss mechanisms, a steady-state system will result, with synchrotron luminosities mirroring the electron injection rates from supernovae. However, the evidence is compelling that in powerful nuclear starbursts like that in Arp 220, supernovae generate local overpressures that drive superwinds. These superwinds are often detectable in X-rays, and evidence for a powerful superwind has been found in Arp 220 itself (Heckman et al. 1996; McDowell et al. 2003). Since the western nucleus exhibits direct evidence for a higher mechanical energy-injection rate per unit volume from supernovae than the eastern nucleus, it is plausible that the pressure is correspondingly higher, and the resulting expansion losses more rapid, compared to the eastern nucleus. Such differential expansion losses would depress the diffuse radio continuum flux density in the west, as observed.

Free-free absorption, synchrotron aging, and expansion losses will all manifest themselves in the spectral behavior of the radio emission from the two nuclei. Detailed high-sensitivity spectral mapping at 0.1 resolution will be needed to further constrain these phenomena in the Arp 220 nuclei. This may soon be possible with the expanded bandwidths and sensitivity of the e-MERLIN instrument.

On a cautionary note, free-free absorption will preferentially depress the flux densities of the RSN in the western nucleus. If these reduced flux densities fall below our detection limit for some new sources up to a year old, the inferred supernova rate in the western nucleus will be depressed relative to that in the east. The only way to discover if our census of new supernovae is complete, and therefore if our inferred supernova rate estimates are unbiased between the two nuclei, is to see if increased imaging sensitivity yields increased rates of appearance of new sources. This will be feasible in coming years as bandwidths for VLBI systems continue to improve.

To summarize our conclusions from this section, our images of RSNe trace an energetic starburst in progress in both Arp 220 nuclei. Regarding the luminosity ratio between the nuclei, comparison of our data with mid-IR imaging data leads to a consistent picture of a 3:1 ratio of west/east luminosities. A starburst origin for the bulk of the Arp 220 bolometric luminosity is supported by this comparison. The failure of the diffuse radio continuum emission to exhibit a similar 3:1 ratio can be understood in terms of differential free-free absorption, and more speculatively, in terms of differential synchrotron plasma expansion losses.

\subsection{Comparison to M82}

The starburst galaxy M82 lies at a distance of only $3.2 \mathrm{Mpc}$, and as such is amenable to detailed study at much lower luminosity levels. It is the only other galaxy in which large numbers of compact sources have been detected. Despite the obvious difference in the scale of the phenomenon, there are striking similarities, and we deem a comparison of the two galaxies to be useful. The far-infrared luminosity of M82 is $2.8 \times 10^{10} L_{\odot}$ (Telesco 1988), which is roughly 50 times lower than that of Arp 220. The star formation rate is therefore expected to be lower by a comparable factor, and with a consequently lower supernova rate, one expects to see a population of supernova remnants that is much older and fainter than that in Arp 220. Qualitatively, this is exactly what is found.

In Figure 3 we show a histogram of compact radio source monochromatic $18 \mathrm{~cm}$ power for M82, and for each of the two nuclei of Arp 220. The detection threshold for Arp 220 is $\sim 10^{19.6} \mathrm{~W} \mathrm{~Hz}^{-1}$, while that for M82 is $\sim 10^{18.0} \mathrm{~W} \mathrm{~Hz}^{-1}$. It is notable that only one of the M82 sources falls above the Arp 220 luminosity detection threshold. If the supernova rate in M82 is 50 times lower than in Arp 220, consistent with the ratio of FIR luminosities, we expect only one RSN in M82 of comparable youth to the 50 that we have detected in Arp 220. In fact, the last RSN appeared in M82 40 years ago, so another is somewhat overdue. The bright M82 source $41.95+57.5$ is decaying at $\sim 8.5 \% \mathrm{yr}^{-1}$, and while it may be a plausible candidate for a young $\mathrm{RSN}$, there is strong evidence that this object is atypical, may not 
be an RSN, and may be much older (Pedlar et al. 1999; McDonald et al. 2001; Beswick et al. 2006). The hypothesis that the starburst in M82 is a scaled-down version of the same phenomenon occurring in Arp 220 is thus attractive, and supported by the data.

Based on this model, we can make an estimate of the number and strength of supernova remnants similar to those observed in M82 that lie below our detection threshold in Arp 220. This number should scale with the FIR luminosity, and the resulting total flux density from 1500 SNRs is $\sim 11 \mathrm{mJy}$, comparable to the total flux density in the 49 detected sources. Based on the observed sizes of the M82 sources (e.g., Muxlow et al. [1994]; McDonald et al. [2001]), the SNRs will have a volume filling factor of up to a few percent in the Arp 220 nuclei. The predicted $11 \mathrm{mJy}$ will contribute to the apparently diffuse emission from the Arp 220 nuclei, but the flux density will actually reside in angularly compact but undetectably weak supernova remnants. This is in addition to the $\sim 12$ mJy of the nuclear flux density that resides in the detected sources. Together, these total $\sim 12 \%$ of the nuclear and $\sim 8 \%$ of the total $18 \mathrm{~cm}$ flux density in Arp 220 . This constitutes a measure of the number of relativistic electrons that remain trapped in high-emissivity regions of supernova remnants, and that have not yet diffused into the lower emissivity environment of the general ISM.

This conclusion must be tempered by the realization that the evolution of older supernova remnants in the dense Arp 220 environment may differ systematically from that occurring in M82.

While the hypothesized weak SNRs may be undetectable in continuum emission, it is possible that they will contribute compact spots of enhanced continuum brightness, which may exhibit $\mathrm{OH}$ maser amplification to detectable levels. Interpretation of $\mathrm{OH}$ maser properties of galaxies such as Arp 220 should take this possibility into account.

\section{CONCLUSIONS}

We have presented a $\lambda 18 \mathrm{~cm}$ VLBI image of the compact sources in Arp 220, with $\sim 1$ pc resolution and unprecedented sensitivity. A robust analysis of the image has yielded detections of 49 unresolved sources, of which 20 lie in the eastern nucleus, and 29 lie in the west. The eastern sources are systematically weaker than those in the west. There is some evidence that, in addition, the shape of the luminosity distributions differs between the two nuclei. Four new sources have been observed to appear over a 12 month period, with one in the east and three in the west.

The interpretation of these sources as radio supernovae ( $\mathrm{RSNe}$ ) appears secure, and is strengthened by the appearance of new sources on a short timescale. The detected RSNe population in Arp 220 spans the upper end of the range of peak $18 \mathrm{~cm}$ radio powers for Type II supernovae. The rate at which new sources are appearing yields an estimate of the supernova rate of $4 \pm 2 \mathrm{yr}^{-1}$. There remains a possibility that this rate is underestimated due to sensitivity limitations. The supernova rate can be related to the star formation rate, and current data imply that star formation activity in Arp 220 is sufficient to power the entire observed farinfrared luminosity of the galaxy.

The differences between the two nuclei of Arp 220 are not yet fully understood. However it is clear that the western nucleus is more compact, and evidence from both IR imaging and the current RSN results indicates that it is $\sim 3$ times more luminous than the eastern nucleus. Differential free-free absorption and expansion losses in the synchrotron plasma may be responsible for the failure of the diffuse continuum emission to exhibit a similar $3: 1$ flux ratio between the nuclei. There appear to be additional differences between the RSN populations in the two nuclei that have yet to be explained. It is expected that the ISM will strongly influence the observed RSN properties, and the ISM may differ between the nuclei. Measurements of free-free absorption to each RSN source via multifrequency VLBI will help to distinguish among possibilities.

A comparison of Arp 220 with the nearby, well-studied starburst galaxy M82 yields a consistent picture with regard to supernovarelated compact radio sources, in which the Arp 220 starburst is simply $\sim 50$ times more luminous, and is confined to a smaller volume. By analogy with M82, roughly $6 \%$ of the apparently diffuse continuum emission from the Arp 220 nuclei may reside in an ensemble of weak, compact supernova remnants. This may have observable consequences for $\mathrm{OH}$ maser emission characteristics.

The National Radio Astronomy Observatory is a facility of the National Science Foundation operated under cooperative agreement by Associated Universities, Inc. The Arecibo Observatory is part of the National Astronomy and Ionosphere Center, which is operated by Cornell University under a cooperative agreement with the National Science Foundation. The European VLBI Network is a joint facility of European, Chinese, South African and other radio astronomy institutes funded by their national research councils. This work was partially supported by NSF grant AST0352953 to Haystack Observatory. We thank Rob Beswick for help in compiling data on M82, and useful comments on the manuscript.

\section{REFERENCES}

Alberdi, A., Colina, L., Torrelles, J. M., Panagia, N., Wilson, A. S., \& Garrington, S. T. 2006, ApJ, 638, 938

Allen, M. L., \& Kronberg, P. P. 1998, ApJ, 502, 218

Baan, W. A., Wood, P. A. D., \& Haschick, A. D. 1982, ApJ, 260, L49

Beswick, R. J., et al. 2006, MNRAS, 369, 1221

Colina, L., Alberdi, A., Torrelles, J. M., Panagia, N., \& Wilson, A. S. 2001, ApJ, 553, L19

Condon, J. J., Huang, Z-P., Yin, Q. F., \& Thuan, T. X. 1991, ApJ, 378, 65

Cram, L., Hopkins, A., Mobasher, B., \& Rowan-Robinson, M. 1998, ApJ, 507, 155

Diamond, P. J. 1995, in ASP Conf. Ser. 82, Very Long Baseline Interferometry and the VLBA NRAO, ed. J. A. Zensus, P. J. Diamond \& P. J. Napier (San Francisco: ASP), 227

Heckman, T. M., Dahlem, M., Eales, S. A., Fabbiano, G., \& Weaver, K. 1996, ApJ, 457, 616

Iwasawa, K., Sanders, D. B., Evans, A. S., Trentham, N., Miniutti, G., \& Spoon, H. W. W. 2005, MNRAS, 357, 565

Kronberg, P. P., Sramek, R. A., Birk, G. T., Dufton, Q. W., Clarke, T. E., \& Allen, M. L. 2000, ApJ, 535, 706

Lonsdale, C. J., Diamond, P. J., Smith, H. E. \& Londsdale, C. J. 1994, Nature, 370,117

. 1998, ApJ, 493, L13

McDonald, A. R., Muxlow, T. W. B., Pedlar, A., Garrett, M. A., Wills, K. A., Garrington, S. T., Diamond, P. J., \& Wilkinson, P. N. 2001, MNRAS, 322, 100

McDowell, J. C., Clements, D. L., Lamb, S. A., Shaked, S., Hearn, N. C., Colina, L., Mundell, C., Borne, K., Baker, A. C., \& Arribas, S. 2003, ApJ, 591, 154

Muxlow, T. W. B., Pedlar, A., Wilkinson, P. N., Axon, D. J., Sanders, E. M., de Bruyn, A. G. 1994, MNRAS, 266, 455

Norris, R. P. 1988, MNRAS, 230, 345

Pedlar, A., Muxlow, T. W. B., Garrett, M. A., Diamond, P. J., Wills, K. A., Wilkinson, P. N., \& Alef, W. 1999, MNRAS, 307, 761

Persic, M., Rephaeli, Y., Braito, V., Cappi, M., Della Ceca, R., Franceschini, A., \& Gruber, D. E. 2004, A\&A, 419, 849

Rodriguez-Rico, C. A., Goss, W. M., Zhao, J.-H., Gomez, Y., \& Anantharamaiah, K. R. 2005, ApJ, 633, 198

Rovilos, E., Diamond, P. J., Lonsdale, C. J., Londsdale, C. J. \& Smith, H. E. 2003, MNRAS, 342, 373

. 2005, MNRAS, 359, 827 
Smith, D. A., Herter, T., \& Haynes, M. P. 1998a, ApJ, 494, 150

Smith, H. E., Lonsdale, C. J. \& Lonsdale, C. J. 1998b, ApJ, 492, 137

Smith, H. E., Lonsdale, C. J., Lonsdale, C. J., \& Diamond, P. J. 1998c, ApJ, 493, L17

Soifer, B. T., Neugebauer, G., Matthews, K., Becklin, E. E., Ressler, M., Werner, M. W., Weinberger, A. J., \& Egami, E. 1999, ApJ, 513, 207

Soifer, B. T., Neugebauer, G., Matthews, K., Egami, E., \& Weinberger, A. J. 2002, AJ, 124, 2980

Soifer, B. T., Sanders, D. B., Madore, B. F., Neugebauer, G., Danielson, G. E., Elias, J. H., Lonsdale, C. J., \& Rice, W. L. 1987, ApJ, 320, 238
Telesco, C. M. 1988, ARA\&A, 26, 343

van Dyk, S.D., Weiler, K.W., Montes, M.J., Sramek, R.A., \& Panagia, N. 2000 Proc. Perspectives on Radio Astronomy: Science with Large Antenna Arrays, ed. M. P. van Haarlem (Dwingeloo: ASTRON), 241

Weiler, K. W., Sramek, R. A., Panagia, N., van der Hulst, J. M., \& Salvati, M. 1986, ApJ, 301, 790

Wills, K. A., Pedlar, A., \& Muxlow, T. W. B. 1998, MNRAS, 298, 347 